\title{
On Four-Dimensional Gradient Shrinking Solitons
}

\section{Lei Ni and Nolan Wallach}

Department of Mathematics, University of California at San Diego, La Jolla, CA 92093

Correspondence to be sent to:lni@math.ucsd.edu

In this paper, we classify the four-dimensional gradient shrinking solitons under certain curvature conditions satisfied by all solitons arising from finite-time singularities of Ricci flow on compact four-manifolds with positive isotropic curvature. As a corollary, we generalize a result of Perelman on three-dimensional gradient shrinking solitons to dimension four.

\section{Introduction}

The goal of this paper is to generalize a result of Perelman on three-dimensional gradient shrinking solitons to dimension four. In his surgery paper, Perelman proved the following statement [20]:

Theorem 1.1. Any $\kappa$-non-collapsed (for some $\kappa>0$ ) complete gradient shrinking soliton $M^{3}$ with bounded positive sectional curvature must be compact.

Combiningwith Hamilton's convergence (or curvature pinching) result [8] (see also [12]) one can conclude that $M^{3}$ must be isometric to a quotient of $\mathbb{S}^{3}$. The reader can find more detailed proof of this result in [3, 13, 15] and Theorem 9.79 of [6]. We refer to [17] for the discussion on the uses of such a result in the study of Ricci flow, 
an alternative proof to the above result, the basic framework for the high-dimensional cases, and a related result in high dimensions.

For four manifolds, in [9], Hamilton proved that for any compact Riemannian manifold with positive curvature operator, the Ricci flow deforms it into a metric of constant curvature. Such a result has been generalized by Chen [4] to manifolds whose curvature operator is 2-positive. (Recently, in a foundational work Böhm and Wilking [1] have generalized this result to all dimensions. However, it is still unknown if there exists any four-dimensional complete gradient shrinking solitons with a positive curvature operator that is not compact. There is a recent development on this using the main theorem of the current paper. Please see http://arxiv.org/abs/0710.5579.)

In [10], Hamilton initiated another important direction, Ricci flow with surgery, and used the method to study the topology of four manifolds with positive isotropic curvature.

Recall from [9] that there is a natural splitting of $\wedge^{2}\left(\mathbb{R}^{4}\right)$ into self-dual and antiself-dual parts and one can write the curvature operator $\mathbf{R}$ as

$$
\mathbf{R}=\left(\begin{array}{cc}
A & B \\
B^{t} & C
\end{array}\right)
$$

according to the decomposition $\wedge^{2}\left(\mathbb{R}^{4}\right)=\wedge_{+} \oplus \wedge_{-}$. (See Section 2 for more details.) The first Bianchi identity implies that $\operatorname{tr}(A)=\operatorname{tr}(C)=\frac{S}{4}$ where $S$ is the scalar curvature. Note that $A$ and $C$ are symmetric. Let $A_{1} \leq A_{2} \leq A_{3}$ and $C_{1} \leq C_{2} \leq C_{3}$ be eigenvalues of $A$ and $C$, respectively. Then $\mathbf{R}$ having positive isotropic curvature amounts to that $A_{1}+A_{2}>0$ and $C_{1}+C_{2}>0$. Let $0 \leq B_{1} \leq B_{2} \leq B_{3}$ be the singular values of $B$.

The main purpose of this article is to show a classification result on the gradient shrinking solitons satisfying a rather weak pinching condition:

$$
\left(\frac{B_{3}^{2}}{\left(A_{1}+A_{2}\right)\left(C_{1}+C_{2}\right)}\right)(x) \leq \exp (a(r(x)+1))
$$

for some $a>0$, where $r(x)$ is the distance function to a fixed point on the manifold. As in [17] we also assume that the curvature tensor satisfies

$$
\left|R_{i j k l}\right|(x) \leq \exp (b(r(x)+1))
$$

for some $b>0$. 
Theorem 1.2. Any four-dimensional complete gradient shrinking soliton with nonnegative curvature operator and positive isotropic curvature satisfying (1.1) and (1.2) is either a quotient of $\mathbb{S}^{4}$ or a quotient of $\mathbb{S}^{3} \times \mathbb{R}$.

Let us first comment on the relationship of our result with the Hamilton's work. In [10], it was shown that on the blow-up limit of any finite-time singularity of Ricci flow on a compact 4-manifold initially with positive isotropic curvature, there exists $\delta>0$ depending only on the initial manifold such that the following pinching estimates hold:

$$
A_{1} \geq \delta A_{3}, \quad C_{1} \geq \delta C_{3}, \quad A_{1} C_{1} \geq B_{3}^{2}
$$

where (again) $0 \leq B_{1} \leq B_{2} \leq B_{3}$ are singular values of $B$. We say that $\mathbf{R}$ has uniformly positive isotropic curvature if (1.3) holds with $A_{1} C_{1}>0$. Note that this implies $\mathbf{R} \geq 0$. In view of the work [10] (see also related work [5]) for the study of the Ricci flow on four manifolds with positive isotropic curvature, it is useful to have a classification of gradient shrinking solitons with uniformly positive isotropic curvature in the sense of (1.3).

On the other hand, in general on a gradient shrinking soliton with positive isotropic curvature, it is not clear to the authors whether or not (1.3) always holds. We say that a Riemannian four-manifold $M$ has weakly uniformly positive isotropic curvature if there exists $\Sigma>0$ such that

$$
\left(\frac{B_{3}^{2}}{\left(A_{1}+A_{2}\right)\left(C_{1}+C_{2}\right)}\right)(x) \leq \Sigma .
$$

By Theorem B2.1 of [10], it is easy to infer that a gradient shrinking soliton with bounded curvature satisfying (1.4) must satisfy

$$
\left(\frac{B_{3}^{2}}{\left(A_{1}+A_{2}\right)\left(C_{1}+C_{2}\right)}\right)(x) \leq \frac{1}{4},
$$

which is a lot stronger than our assumption (1.1), but weaker than (1.3).

The use of the classification result is that it rules out the possible complications caused by the existence of noncompact singularity models and implies a classification of finite-time singularities models, which then makes surgery procedure possible. More precisely, ancient solutions, which are noncompact in interesting cases, can be obtained as the Cheeger-Gromov limit of the sequence of blow-ups, via the compactness result of Hamilton along with the no local collapsing theorem of Perelman, as we approach to the singular time. By the pinching result Theorem B1.1 of Hamilton [10], we know that such ancient solution has bounded non-negative curvature operator with 
(1.3). The gradient shrinking solitons (called asymptotic solitons) arise from the noncollapsed ancient solutions as the blow-down limits [19] as $t \rightarrow-\infty$, at least in the case that the ancient solution has non-negative curvature operator. The classification result concludes that the shrinking soliton arisen from the ancient solutions must be cylinder $\mathbb{S}^{3} \times \mathbb{R}$ or its quotient (which can be ruled out under some further topological assumptions/considerations). This provides the phototype for the surgery. (In [10], a different approach is taken to detect the cylinders.) Summarizing, the asymptotic soliton arising from the singularity of Ricci flow on a four-manifold with positive isotropic curvature, has non-negative curvature operator, satisfies (1.3), ensured by the previously mentioned result of Hamilton [10]. Moreover, by [16], the curvature of the asymptotic soliton has at most quadratic growth. Hence Theorem 1.2 does give a complete classification on the asymptotic solitons.

Corollary 1.3. Any asymptotic soliton arising from the finite-time singularities of Ricci flow on a compact manifold initially with positive isotropic curvature is either a quotient of $\mathbb{S}^{4}$ or a quotient of $\mathbb{S}^{3} \times \mathbb{R}$.

We should also remark that in view of the examples [2, 7, 14] some conditions on the curvature operator are essential to obtain a classification result as presented in the preceding paragraphs. As a corollary of Theorem 1.2, we have the following fourdimensional analogue of Theorem 1.1.

Corollary 1.4. Any four-dimensional gradient shrinking soliton with positive curvature operator satisfying (1.1) and (1.2) must be compact.

Note that there exists a general compactness result [17] under a certain pinching condition on the curvature operator, provided that the curvature operator is bounded. But the condition (1.1) is a much weaker one since the curvature operator pinching of [18] implies that there exists $\epsilon>0$ with $A_{1} \geq \epsilon A_{3}, C_{1} \geq \epsilon C_{3}$, which further implies $\left(A_{1}+A_{2}\right)\left(C_{1}+C_{2}\right) \geq \epsilon^{\prime} S^{2} \geq \epsilon^{\prime} \delta$ for some positive $\epsilon^{\prime}$ and $\delta$ (by Proposition 1.1 of [16]). Here (again) $S$ denotes the scalar curvature. From the last estimate and the boundedness of curvature, one can deduce (1.4).

\section{Preliminary Result}

By [9], the curvature operator $\mathbf{R}$ satisfies the equation

$$
\left(\frac{\partial}{\partial t}-\Delta\right) \mathbf{R}=2 \mathbf{R}^{2}+2 \mathbf{R}^{\#}
$$


where $\mathbf{R}^{2}$ is defined as transformations of $\wedge^{2}\left(\mathbb{R}^{n}\right)$, and $\mathbf{R}^{\#}$ is defined via the standard Lie algebra structure on $\wedge^{2}\left(\mathbb{R}^{n}\right)$. The reader should consult [9] for details. In dimension four, $\wedge^{2}\left(\mathbb{R}^{4}\right)$ decomposes into self-dual part $\wedge_{+}$and anti-self-dual part $\wedge_{-}$. This leads to the decomposition of $\mathbf{R}$ mentioned in Section 1. For the following computation, we may choose the basis for $\wedge_{+}$and $\wedge_{-}$as

$$
\begin{aligned}
& \eta_{1}=\frac{1}{\sqrt{2}}\left(e_{1} \wedge e_{2}+e_{3} \wedge e_{4}\right), \quad \xi_{1}=\frac{1}{\sqrt{2}}\left(e_{1} \wedge e_{2}-e_{3} \wedge e_{4}\right) \\
& \eta_{2}=\frac{1}{\sqrt{2}}\left(e_{1} \wedge e_{3}+e_{4} \wedge e_{2}\right), \quad \xi_{2}=\frac{1}{\sqrt{2}}\left(e_{1} \wedge e_{3}-e_{4} \wedge e_{2}\right) \\
& \eta_{3}=\frac{1}{\sqrt{2}}\left(e_{1} \wedge e_{4}+e_{2} \wedge e_{3}\right), \quad \xi_{3}=\frac{1}{\sqrt{2}}\left(e_{1} \wedge e_{4}-e_{2} \wedge e_{3}\right)
\end{aligned}
$$

where $\left\{e_{1}, e_{2}, e_{3}, e_{4}\right\}$ is a positively oriented basis. From [9] we know that

$$
\mathbf{R}^{\#}=2\left(\begin{array}{cc}
A^{\#} & B^{\#} \\
\left(B^{t}\right)^{\#} & C^{\#}
\end{array}\right)
$$

the traceless part of $A$ and $C$ are $W_{+}$and $W_{-}$, the self-dual part and the anti-self-dual part of Weyl curvature, and $B$ is the traceless Ricci curvature. It is easy to see that $\operatorname{tr}(A)=\operatorname{tr}(C)=\frac{S}{4}$. Here $S$ is the scalar curvature. Notice that $A^{\#}, B^{\#}, C^{\#}$ are computed as transformations of $\wedge^{2}\left(\mathbb{R}^{3}\right)$. For example, $A^{\#}=\operatorname{det}(A)\left(A^{t}\right)^{-1}$, while $B^{\#}=-\operatorname{det}(B)\left(B^{t}\right)^{-1}$.

Let $\sigma^{2}=|\operatorname{Ric}|^{2}$ and $\tilde{\sigma}^{2}=\left|\operatorname{Ric}_{0}\right|^{2}$, where $\operatorname{Ric}_{0}$ is the traceless part of Ricci tensor. Also, let $\lambda_{i}$ be the eigenvalue of Ric $_{0}$. First we shall determine how $\tilde{\sigma}^{2}$ is related to $B$. Direct computation shows that

$$
B=\frac{1}{2}\left(\begin{array}{ccc}
R_{1212}-R_{3434} & R_{23}-R_{14} & R_{24}+R_{13} \\
R_{23}+R_{14} & R_{1313}-R_{2424} & R_{34}-R_{12} \\
R_{24}-R_{13} & R_{34}+R_{12} & R_{1414}-R_{2323}
\end{array}\right)
$$

Here $R_{i j}$ are the Ricci tensor components. From this we have the following expression of Ric $_{0}$ in terms of $B$ :

$$
\operatorname{Ric}_{0}=\left(\begin{array}{cccc}
B_{11}+B_{22}+B_{33} & B_{32}-B_{23} & B_{13}-B_{31} & B_{21}-B_{12} \\
B_{32}-B_{23} & B_{11}-B_{22}-B_{33} & B_{21}+B_{12} & B_{13}+B_{31} \\
B_{13}-B_{31} & B_{21}+B_{12} & B_{22}-B_{11}-B_{33} & B_{23}+B_{32} \\
B_{21}-B_{12} & B_{13}+B_{31} & B_{23}+B_{32} & B_{33}-B_{11}-B_{22}
\end{array}\right) .
$$


Direct computation shows that

$$
\tilde{\sigma}^{2}=4|B|^{2} \quad \text { and } \quad \sum_{1}^{4} \lambda_{j}^{3}=-8 \operatorname{tr}\left(B^{\#} B^{t}\right)
$$

Now define

$$
P \doteqdot 2 \operatorname{tri}(\mathbf{R}) S-\sigma^{2}\left|R_{i j k l}\right|^{2}
$$

where $\operatorname{tri}(\mathbf{R})=2\left\langle\mathbf{R}^{2}+\mathbf{R}^{\#}, \mathbf{R}\right\rangle$. In [17], it was shown that

$$
\left(\frac{\partial}{\partial t}-\Delta\right)\left(\frac{\left|R_{i j k l}\right|^{2}}{S^{2}}\right)=\frac{4 P}{S^{3}}-\frac{2}{S^{4}}\left|S \nabla_{p} R_{i j k l}-\nabla_{p} S R_{i j k l}\right|^{2}+\left\langle\nabla\left(\frac{\left|R_{i j k l}\right|^{2}}{S^{2}}\right), \nabla \log S^{2}\right\rangle .
$$

By the proof of the main theorem in [17], the classification result follows from the nonpositivity of $P$. We now compute it in terms of $A, B, C$. First, it is easy to see that

$$
P=4\left\langle S\left(\mathbf{R}^{2}+\mathbf{R}^{\#}\right)-\left(\frac{S^{2}}{n}+\tilde{\sigma}^{2}\right) \mathbf{R}, \mathbf{R}\right\rangle .
$$

For the case $\operatorname{dim}(M)=4$ we have that

$$
\begin{aligned}
\left\langle\mathbf{R}^{2}+\mathbf{R}^{\#}, \mathbf{R}\right\rangle= & \operatorname{tr}\left(A^{3}\right)+\operatorname{tr}\left(C^{3}\right)+2 \operatorname{tr}\left(A^{\#} A\right)+2 \operatorname{tr}\left(\left(B^{t}\right)^{\#} B\right)+2 \operatorname{tr}\left(B^{\#} B^{t}\right) \\
& +2 \operatorname{tr}\left(C^{\#} C\right)+3 \operatorname{tr}\left(A B B^{t}\right)+3 \operatorname{tr}\left(C B^{t} B\right)
\end{aligned}
$$

and

$$
\langle\mathbf{R}, \mathbf{R}\rangle=\operatorname{tr}\left(A^{2}\right)+\operatorname{tr}\left(C^{2}\right)+2|B|^{2} .
$$

Hence

$$
\begin{aligned}
\frac{1}{4} P= & S\left(\operatorname{tr}\left(A^{3}\right)+\operatorname{tr}\left(C^{3}\right)+2 \operatorname{tr}\left(A^{\#} A+C^{\#} C\right)+2 \operatorname{tr}\left(\left(B^{t}\right)^{\#} B\right)+2 \operatorname{tr}\left(B^{\#} B^{t}\right)+3 \operatorname{tr}\left(A B B^{t}\right)\right. \\
& \left.+3 \operatorname{tr}\left(C B^{t} B\right)\right)-\left(\frac{S^{2}}{4}+4|B|^{2}\right)\left(\operatorname{tr}\left(A^{2}\right)+\operatorname{tr}\left(C^{2}\right)+2|B|^{2}\right)
\end{aligned}
$$

Let $\stackrel{o}{A}$ be the traceless part of $A$. Similarly, we have $\stackrel{o}{C}$. By choosing suitable basis we may diagonalize $\stackrel{\circ}{A}$ and $\stackrel{O}{C}$ such that we can assume that

$$
A=\left(\begin{array}{ccc}
\frac{S}{12}+a_{1} & 0 & 0 \\
0 & \frac{S}{12}+a_{2} & 0 \\
0 & 0 & \frac{S}{12}+a_{3}
\end{array}\right), \quad C=\left(\begin{array}{ccc}
\frac{S}{12}+c_{1} & 0 & 0 \\
0 & \frac{S}{12}+c_{2} & 0 \\
0 & 0 & \frac{S}{12}+c_{3}
\end{array}\right)
$$


Now we can write

$$
\begin{aligned}
P= & -S^{2}\left(\frac{1}{6} \sum_{1}^{4} \lambda_{i}^{2}+\sum_{1}^{3} a_{i}^{2}+\sum_{1}^{3} c_{i}^{2}\right)+4 S\left(\sum_{1}^{3}\left(a_{i}^{3}+c_{i}^{3}\right)+6 a_{1} a_{2} a_{3}+6 c_{1} c_{2} c_{3}-\frac{1}{2} \sum_{1}^{4} \lambda_{i}^{3}\right) \\
& +12 S\left(a_{1} b_{1}^{2}+a_{2} b_{2}^{2}+a_{3} b_{3}^{2}+c_{1} \tilde{b}_{1}^{2}+c_{2} \tilde{b}_{2}^{2}+c_{3} \tilde{b}_{3}^{2}\right) \\
& -2\left(\sum_{1}^{4} \lambda_{i}^{2}\right)^{2}-4\left(\sum_{i=1}^{4} \lambda_{i}^{2}\right)\left(\sum_{i=1}^{3}\left(a_{i}^{2}+c_{i}^{2}\right)\right) .
\end{aligned}
$$

Here $\sum_{1}^{3} a_{i}=\sum_{1}^{3} c_{i}=\sum_{1}^{4} \lambda_{j}=0, b_{i}^{2}=\sum_{j=1}^{3} B_{i j}^{2}$ and $\tilde{b}_{i}^{2}=\sum_{j=1}^{3} B_{j i}^{2}$. Hence $\sum_{1}^{3} b_{i}^{2}=\sum_{1}^{3} \tilde{b}_{i}^{2}=$ $\frac{1}{4} \sum_{1}^{4} \lambda_{j}^{2}$

We first consider a few basic examples. Differing by some scaling constants (since the sign of $P$ is independent of the scaling), we have that

$$
\mathbf{R}_{\mathbb{S}^{4}}=\left(\begin{array}{cc}
\text { id } & 0 \\
0 & \text { id }
\end{array}\right), \quad \mathbf{R}_{\mathbb{S}^{3} \times \mathbb{R}}=\left(\begin{array}{cc}
\text { id } & F \\
F^{t} & \text { id }
\end{array}\right), \quad \mathbf{R}_{\mathbb{S}^{2} \times \mathbb{S}^{2}}=\left(\begin{array}{cc}
E & 0 \\
0 & E
\end{array}\right), \quad \mathbf{R}_{\mathbb{S}^{2} \times \mathbb{R}^{2}}=\left(\begin{array}{cc}
E & E \\
E & E
\end{array}\right)
$$

where

$$
F=\left(\begin{array}{ccc}
1 & 0 & 0 \\
0 & 1 & 0 \\
0 & 0 & -1
\end{array}\right), \quad E=\left(\begin{array}{lll}
1 & 0 & 0 \\
0 & 0 & 0 \\
0 & 0 & 0
\end{array}\right)
$$

It is easy to check that $P=0$ in these examples. On the complex projective space,

$$
\mathbf{R}_{\mathbb{P}^{2}}=\left(\begin{array}{cc}
\mathrm{id} & 0 \\
0 & 3 E
\end{array}\right)
$$

Again $P=0$ !

With suitable choices of the orthornormal basis for $\Lambda_{+}$and $\Lambda_{-}$we can assume that $A_{i}=\frac{S}{12}+a_{i}, C_{i}=\frac{S}{12}+c_{i}$. It is easy to see that $\max \left\{\tilde{b}_{i}^{2}, b_{i}^{2}\right\} \leq B_{3}^{2}$ for any $1 \leq i \leq 3$.

The main result of this section is to prove a special case of Theorem 1.2.

Proposition 2.1. Suppose that $B B^{t}=b^{2}$ id for some $b, A$ and $C$ are positive semidefinite. Then $P \leq 0$ and the universal cover of $M$ is either $\mathbb{S}^{4}$ or $\mathbb{S}^{3} \times \mathbb{R}$.

Proof. Observing that

$$
\sum a_{i}^{3}+6 a_{1} a_{2} a_{3}=3 \sum a_{i}^{3}
$$

in order to show that $2 \operatorname{tri}(\mathbf{R}) S-\sigma^{2}\left|R_{i j k l}\right|^{2} \leq 0$ it suffices to show that

$$
-S^{2} \sum a_{i}^{2}+12 S \sum a_{i}^{3}-48 b^{2} \sum a_{i}^{2} \leq 0
$$


since the same argument also proves the same statement for the same expressions involving $c_{i}$. Here we have used that $\sum \lambda_{i}^{2}=12 b^{2}$. Note that we have the constraints that $\frac{S}{12}+a_{i} \geq 0$ and $\sum a_{i}=0$. Using the fact that $\sum a_{i}^{3} \leq \frac{1}{\sqrt{6}}$ under the constraints $\sum a_{i}=0$ and $\sum a_{i}^{2}=1$, which can be obtained by Proposition 5.1 of [17], we conclude that

$$
\frac{\sum a_{i}^{3}}{\sum a_{i}^{2}} \leq \frac{1}{\sqrt{6}} a
$$

where $a^{2}=\sum a_{i}^{2}$. (This fact can also be established directly using the Lagrange multipliers.) Moreover, the fact thatthe equality holds implies that $a_{i}=0$ for $1 \leq i \leq 3$ or $a_{1}=a_{2}=-\frac{1}{\sqrt{6}} a$ and $a_{3}=\sqrt{\frac{2}{3}} a$. Equivalently,

$$
A=\left(\begin{array}{ccc}
\frac{S}{12} & 0 & 0 \\
0 & \frac{S}{12} & 0 \\
0 & 0 & \frac{S}{12}
\end{array}\right) \quad \text { or } \quad\left(\begin{array}{ccc}
\frac{S}{12}-\frac{1}{\sqrt{6}} a & 0 & 0 \\
0 & \frac{S}{12}-\frac{1}{\sqrt{6}} a & 0 \\
0 & 0 & \frac{S}{12}+\sqrt{\frac{2}{3}} a
\end{array}\right)
$$

On the other hand, under the constraints $\frac{S}{12}+a_{i} \geq 0$ and $\sum a_{i}=0$, the maximum of $\sum a_{i}^{2}$ is $\frac{S^{2}}{24}$, which can be better seen by expressing everything in terms of $A_{i}=$ $\frac{S}{12}+a_{i} \geq 0$. This shows that $-S^{2} \sum a_{i}^{2}+12 S \sum a_{i}^{3} \leq 0$ in view of $S>0$. We can handle the terms with $c_{i}$ 's similarly. Furthermore, $-S^{2} \sum a_{i}^{2}+12 S \sum a_{i}^{3}-48 b^{2} \sum a_{i}^{2}=S^{2} \sum c_{i}^{2}+$ $12 S \sum c_{i}^{3}-48 b^{2} \sum c_{i}^{2}=0$ implies either $b=0$ and $a_{3}=\frac{S}{6}, a_{1}=a_{2}=-\frac{S}{12}$, or $a_{i}=0$, and correspondingly $b=0$ and $c_{3}=\frac{S}{6}, c_{1}=c_{2}=-\frac{S}{12}$, or $c_{i}=0$. The case with $a_{i}=c_{i}=0$ is of locally conformally flat. The cases that are not locally conformally flat have that

$$
A=\left(\begin{array}{ccc}
0 & 0 & 0 \\
0 & 0 & 0 \\
0 & 0 & \frac{S}{4}
\end{array}\right) \quad \text { or } \quad C=\left(\begin{array}{ccc}
0 & 0 & 0 \\
0 & 0 & 0 \\
0 & 0 & \frac{S}{4}
\end{array}\right)
$$

Hence they are excluded by the positivity of the isotropic curvature. The locally conformally flat case was reduced to the previous result of authors in [17]. Invoking the proof of Corollary 5.2 of [17], we obtain a complete classification for this special case. Note that we have used that $A$ and $C$ are semipositive definite to ensure that $\max \left\{\sum a_{i}^{2}, \sum c_{i}^{2}\right\} \leq \frac{S^{2}}{24}$.

In Section 3 we shall reduce the proof of Theorem 1.2 to this special case.

Remark 2.2. It was pointed out to us by Christoph Böhm, via an explicit example, that the method of this section of considering the evolution equation on $\frac{|\mathbf{R}|^{2}}{S^{2}}$ is not sufficient to obtain the classification result for gradient shrinking solitons with positive curvature operator in dimension four, unlike the three-dimensional cases treated in [17]. 


\section{Proof}

First we observe that some of the ordinary differential inequalities in [9] also hold as partial differential inequalities. We list the ones needed below.

Proposition 3.1. Let $(M, g(t))$ be a solution to Ricci flow. Let $A_{i}, B_{i}$, and $C_{i}$ be the components of curvature operator as defined in the first section. Then

$$
\begin{gathered}
\left(\frac{\partial}{\partial t}-\Delta\right)\left(A_{1}+A_{2}\right) \geq A_{1}^{2}+A_{2}^{2}+2\left(A_{1}+A_{2}\right) A_{3}+B_{1}^{2}+B_{2}^{2} \\
\left(\frac{\partial}{\partial t}-\Delta\right)\left(C_{1}+C_{2}\right) \geq C_{1}^{2}+C_{2}^{2}+2\left(C_{1}+C_{2}\right) C_{3}+B_{1}^{2}+B_{2}^{2} \\
\left(\frac{\partial}{\partial t}-\Delta\right) B_{3} \leq A_{3} B_{3}+C_{3} B_{3}+2 B_{1} B_{2} .
\end{gathered}
$$

The differential inequality can be understood in the sense of distributions.

Proof. The proof is essentially a repeat of the methods used in [10]. Using a timedependent moving frame, we have that

$$
\left(\frac{\partial}{\partial t}-\Delta\right) \mathbf{R}=\mathbf{R}^{2}+\mathbf{R}^{\#}
$$

(The careful reader may notice that there is a factor of 2 difference between this equation and (2.1) used in Section 2. This factor can be easily absorbed by a reparameterization of the time variable and does not affect anything in the argument. We use this simple form as in [9] to avoid keeping track of the extra universal constants.) Fix a point $\left(x_{0}, t_{0}\right)$, choose a local frame so that $A$ and $C$ are diagonal at $x_{0}$. Notice that $\sum_{i, j=1}^{2} A_{i j} g^{i j} \geq A_{1}+A_{2}$ and equality holds at $\left(x_{0}, t_{0}\right)$. Hence at $\left(x_{0}, t_{0}\right)$ we have that

$$
\begin{aligned}
\left(\frac{\partial}{\partial t}-\Delta\right)\left(\sum_{i, j=1}^{2} A_{i j} g^{i j}\right) & =\sum_{i, j=1}^{2} g^{i j}\left(A^{2}+B B^{t}+2 A^{\#}\right)_{i j} \\
& \geq A_{1}^{2}+A_{2}^{2}+2\left(A_{1}+A_{2}\right) A_{3}+B_{1}^{2}+B_{2}^{2} .
\end{aligned}
$$

In the last line we used that $\sum_{i, j=1}^{2} g^{i j}\left(A^{2}\right)_{i j} \geq A_{1}^{2}+A_{2}^{2}, \sum_{i, j=1}^{2} g^{i j}\left(B B^{t}\right)_{i j} \geq B_{1}^{2}+B_{2}^{2}$, as well as the fact that at $\left(x_{0}, t_{0}\right), A$ is diagonal and $A^{\#}$ is diagonal with eigenvalue $A_{2} A_{3}$ and $A_{1} A_{3}$. This shows the partial differential inequality in the sense of barrier. The reader can also find detailed elaborations in [22], Theorem 5.3, for this rather elementary fact. It then follows from the PDE theory, in viewing of the concavity of $A_{1}+A_{2}$, that the inequality 
also holds in the sense of distribution (see for example [11]). The other two inequalities can be shown similarly.

Now we let $\psi_{1}=A_{1}+A_{2}, \psi_{2}=C_{1}+C_{2}, \varphi=B_{3}$. Our assumption on $M$ has positive isotropic curvature implies that $\psi_{1}>0, \psi_{2}>0$. In the computations below we also assume $B_{3}>0$. But it will be clear later on that this is not necessary. Proposition 3.1 together with some straightforward computations implies

$$
\begin{aligned}
\left(\frac{\partial}{\partial t}-\Delta\right) \log \left(\frac{\varphi^{2}}{\psi_{1} \psi_{2}}\right) \leq & 2|\nabla \log \varphi|^{2}-\left|\nabla \log \psi_{1}\right|^{2}-\left|\nabla \log \psi_{2}\right|^{2}-\frac{4 B_{1}\left(B_{3}-B_{2}\right)}{B_{3}} \\
& -\frac{\left(A_{1}-B_{1}\right)^{2}+\left(A_{2}-B_{2}\right)^{2}+2 A_{2}\left(B_{2}-B_{1}\right)}{A_{1}+A_{2}} \\
& -\frac{\left(C_{1}-B_{1}\right)^{2}+\left(C_{2}-B_{2}\right)^{2}+2 C_{2}\left(B_{2}-B_{1}\right)}{C_{1}+C_{2}} .
\end{aligned}
$$

Let

$$
\begin{aligned}
-E= & -\frac{4 B_{1}\left(B_{3}-B_{2}\right)}{B_{3}}-\frac{\left(A_{1}-B_{1}\right)^{2}+\left(A_{2}-B_{2}\right)^{2}+2 A_{2}\left(B_{2}-B_{1}\right)}{A_{1}+A_{2}} \\
& -\frac{\left(C_{1}-B_{1}\right)^{2}+\left(C_{2}-B_{2}\right)^{2}+2 C_{2}\left(B_{2}-B_{1}\right)}{C_{1}+C_{2}} .
\end{aligned}
$$

It is clear that $-E \leq 0$ with equality holds only if $A_{1}=C_{1}=B_{1}=B_{2}=A_{2}=C_{2}=B_{3}$. In particular we have that $B_{1}=B_{2}=B_{3}$, namely $B B^{t}=b^{2}$ id. Using this partial differential inequality, we have that

$$
\begin{aligned}
\left(\frac{\partial}{\partial t}-\Delta\right)\left(\frac{\varphi^{2}}{\psi_{1} \psi_{2}}\right)^{2} \leq & \left(\frac{\varphi^{2}}{\psi_{1} \psi_{2}}\right)^{2}\left(4|\nabla \log \varphi|^{2}-2\left|\nabla \log \psi_{1}\right|^{2}-2\left|\nabla \log \psi_{2}\right|^{2}-2 E\right) \\
& -4\left(\frac{\varphi^{2}}{\psi_{1} \psi_{2}}\right)^{2}\left|2 \nabla \log \varphi-\nabla \log \psi_{1}-\nabla \log \psi_{2}\right|^{2} .
\end{aligned}
$$

Now we compute the gradient terms.

$$
\begin{aligned}
4 \mid \nabla & \left.\log \varphi\right|^{2}-2\left|\nabla \log \psi_{1}\right|^{2}-2\left|\nabla \log \psi_{2}\right|^{2}-4\left|2 \nabla \log \varphi-\nabla \log \psi_{1}-\nabla \log \psi_{2}\right|^{2} \\
= & -2\left|2 \nabla \log \varphi-\nabla \log \psi_{1}-\nabla \log \psi_{2}\right|^{2}+2\left\langle\nabla \log \frac{\varphi}{\psi_{1}}, \nabla \log \left(\varphi \psi_{1}\right)\right\rangle \\
& +2\left\langle\nabla \log \frac{\varphi}{\psi_{2}}, \nabla \log \left(\varphi \psi_{2}\right)\right\rangle-2\left\langle\nabla \log \frac{\varphi}{\psi_{1}}, \nabla \log \frac{\varphi}{\psi_{1}}\right\rangle \\
& -2\left\langle\nabla \log \frac{\varphi}{\psi_{2}}, \nabla \log \frac{\varphi}{\psi_{2}}\right\rangle-4\left\langle\nabla \log \frac{\varphi}{\psi_{1}}, \nabla \log \frac{\varphi}{\psi_{2}}\right\rangle
\end{aligned}
$$




$$
\begin{aligned}
= & -2\left|\nabla \log \frac{\varphi}{\psi_{1}}+\nabla \log \frac{\varphi}{\psi_{2}}\right|^{2}+8\left\langle\nabla \log \varphi, \nabla \log \psi_{1}\right\rangle+8\left\langle\nabla \log \varphi, \nabla \log \psi_{2}\right\rangle \\
& -4\left|\nabla \log \psi_{1}\right|^{2}-4\left|\nabla \log \psi_{2}\right|^{2}-4|\nabla \log \varphi|^{2}-4\left\langle\nabla \log \psi_{1}, \nabla \log \psi_{2}\right\rangle \\
= & -2\left|\nabla \log \frac{\varphi}{\psi_{1}}+\nabla \log \frac{\varphi}{\psi_{2}}\right|^{2}-2\left|\nabla \log \frac{\varphi}{\psi_{1}}\right|^{2}-2\left|\nabla \log \frac{\varphi}{\psi_{2}}\right|^{2} \\
& +2\left\langle\nabla \log \frac{\varphi^{2}}{\psi_{1} \psi_{2}}, \nabla\left(\log \psi_{1} \psi_{2}\right)\right\rangle .
\end{aligned}
$$

Putting all of these calculations together we have that

$$
\begin{aligned}
\left(\frac{\partial}{\partial t}-\Delta\right)\left(\frac{\varphi^{2}}{\psi_{1} \psi_{2}}\right)^{2} \leq & -2\left(\frac{\varphi^{2}}{\psi_{1} \psi_{2}}\right)^{2} E+\left\langle\nabla\left(\frac{\varphi^{2}}{\psi_{1} \psi_{2}}\right)^{2}, \nabla\left(\log \psi_{1} \psi_{2}\right)\right\rangle \\
& -2\left(\frac{\varphi^{2}}{\psi_{1} \psi_{2}}\right)^{2}\left(\left|\nabla \log \frac{\varphi}{\psi_{1}}+\nabla \log \frac{\varphi}{\psi_{2}}\right|^{2}+\left|\nabla \log \frac{\varphi}{\psi_{1}}\right|^{2}+\left|\nabla \log \frac{\varphi}{\psi_{2}}\right|^{2}\right) .
\end{aligned}
$$

It is clear that the right hand side of the above inequality can be rewritten so that $\varphi>0$ is not really required since $\varphi^{2} \nabla \log \varphi=\varphi \nabla \varphi$. Since $(M, g)$ is a gradient shrinking soliton, letting $f$ be the potential function, the computation in the Section 1 of [17] implies that

$$
\frac{\partial}{\partial t}\left(\frac{\varphi^{2}}{\psi_{1} \psi_{2}}\right)^{2}=\left\langle\nabla f, \nabla\left(\frac{\varphi^{2}}{\psi_{1} \psi_{2}}\right)^{2}\right\rangle .
$$

In fact, on a gradient soliton $(M, g(t), f)$, if a scalar function $h(x, t)$ is obtained by pulling back a function at time $t=-1$ via the one-parameter family of diffeomorphisms generated by the vector field $\nabla f$, it holds that $\frac{\partial h}{\partial t}=\langle\nabla f, \nabla h\rangle$. This is quite clear from the proof of Theorem 4.1 of [6]. Now multiply both sides of (3.1) by $e^{-f+\log \left(\psi_{1} \psi_{2}\right)}$ and integrate over the manifold:

$$
\begin{aligned}
\int_{M} & \left\langle\nabla f, \nabla\left(\frac{\varphi^{2}}{\psi_{1} \psi_{2}}\right)^{2}\right\rangle e^{-f+\log \left(\psi_{1} \psi_{2}\right)}-\int_{M}\left(\Delta\left(\frac{\varphi^{2}}{\psi_{1} \psi_{2}}\right)^{2}\right) e^{-f+\log \left(\psi_{1} \psi_{2}\right)} \\
\leq & -2 \int_{M}\left(\frac{\varphi^{2}}{\psi_{1} \psi_{2}}\right)^{2} E e^{-f+\log \left(\psi_{1} \psi_{2}\right)}+\int_{M}\left\langle\nabla\left(\frac{\varphi^{2}}{\psi_{1} \psi_{2}}\right)^{2}, \nabla\left(\log \psi_{1} \psi_{2}\right)\right\rangle e^{-f+\log \left(\psi_{1} \psi_{2}\right)} \\
& -2 \int_{M}\left(\frac{\varphi^{2}}{\psi_{1} \psi_{2}}\right)^{2}\left(\left|\nabla \log \frac{\varphi}{\psi_{1}}+\nabla \log \frac{\varphi}{\psi_{2}}\right|^{2}\right) e^{-f+\log \left(\psi_{1} \psi_{2}\right)} \\
& -2 \int_{M}\left(\frac{\varphi^{2}}{\psi_{1} \psi_{2}}\right)^{2}\left(\left|\nabla \log \frac{\varphi}{\psi_{1}}\right|^{2}+\left|\nabla \log \frac{\varphi}{\psi_{2}}\right|^{2}\right) e^{-f+\log \left(\psi_{1} \psi_{2}\right)}
\end{aligned}
$$


All the integrals are finite by the derivative estimates of Shi [21], the assumption (1.1) and (1.2), and Lemma 1.3 of [17] asserting that $f(x) \geq \frac{1}{8} r^{2}(x)-C$ (with $C>0$ and $r(x)$ being distance function to a fixed point). The assumption (1.2) is needed to ensure a pointwise growth estimate on the derivative of the curvature, more precisely $\left|\nabla \psi_{i}\right|$ and $|\nabla \varphi|$. The finiteness of the integral is due to the integrability of function $e^{-\frac{1}{8} r^{2}(x)+b r(x)+a}$ for any positive constant $a$ and $b$ on $M$. One can consult [17] for more details. As in [17], integration by parts can be performed on the term involving the Laplacian operator on the left-hand side of the preceding inequality. After the integration by parts and some cancelations, we have that

$$
\begin{aligned}
0 \leq & -\int_{M}\left(\frac{\varphi^{2}}{\psi_{1} \psi_{2}}\right)^{2} E e^{-f+\log \left(\psi_{1} \psi_{2}\right)}-2 \int_{M}\left(\frac{\varphi^{2}}{\psi_{1} \psi_{2}}\right)^{2}\left(\left|\nabla \log \frac{\varphi}{\psi_{1}}+\nabla \log \frac{\varphi}{\psi_{2}}\right|^{2}\right) e^{-f+\log \left(\psi_{1} \psi_{2}\right)} \\
& -2 \int_{M}\left(\frac{\varphi^{2}}{\psi_{1} \psi_{2}}\right)^{2}\left(\left|\nabla \log \frac{\varphi}{\psi_{1}}\right|^{2}+\left|\nabla \log \frac{\varphi}{\psi_{2}}\right|^{2}\right) e^{-f+\log \left(\psi_{1} \psi_{2}\right)}
\end{aligned}
$$

which implies that

$$
E=\left|\nabla \log \frac{\varphi}{\psi_{1}}+\nabla \log \frac{\varphi}{\psi_{2}}\right|=\left|\nabla \log \frac{\varphi}{\psi_{1}}\right|=\left|\nabla \log \frac{\varphi}{\psi_{2}}\right|=0 .
$$

In particular, we conclude that $B B^{t}=b^{2} \mathrm{id}$.

\section{Acknowledgments}

The first author would like to thank Christoph Böhm and Burkhard Wilking for helpful discussions and suggestions. We also thank the referees for their numerous comments, which have greatly enhanced the readability of the paper. The first author's research was supported in part by NSF grant DMS-0504792 and an Alfred P. Sloan Fellowship, USA. The second author's research was partially supported by an NSF grant DMS-0500495.

\section{References}

[1] Böhm, C., and B. Wilking. "Manifolds with positive curvature operator are space form." Annals of Mathematics (forthcoming).

[2] Cao, H.-D. "Existence of gradient Kähler-Ricci solitons." Elliptic and Parabolic Methods in Geometry, 1-16. Wellesley, MA: A K Peters, 1996.

[3] Cao, H.-D., and X.-P. Zhu. "A complete proof of the Poincar and geometrization conjecturesapplication of the Hamilton-Perelman theory of the Ricci flow." Asian Journal of Mathematics 10, no. 2 (2006): 165-492.

[4] Chen, H. "Point-wise 1/4-pinched 4-manifolds." Annals of Global Analysis and Geometry 9 (1991): 161-76. 
[5] Chen, B.-L., and X.-P. Zhu. "Ricci flow with surgery on four-manifolds with positive isotropic curvature." Journal of Differential Geometry 74 (2006): 177-264.

[6] Chow, B., P. Lu, and L. Ni. "Hamilton's Ricci flow." Graduate Studies in Mathematics, vol. 77, xxxvi+608. Providence, RI: American Mathematical Society, 2006.

[7] Feldman, M., T. Ilmanen, and D. Knopf. "Rotationally symmetric shrinking and expanding gradient Kähler-Ricci solitons." Journal of Differential Geometry 65, no. 2 (2003): 169-209.

[8] Hamilton, R. "Three-manifolds with positive Ricci curvature." Journal of Differential Geometry 17, no. 2 (1982): 255-306.

[9] Hamilton, R. "Four-manifolds with positive curvature operator." Journal of Differential Geometry 24 (1986): 153-79.

[10] Hamilton, R. "Four-manifolds with positive isotropic curvature." Communications in Analytical Geometry 5, no. 1 (1997): 1-92.

[11] Ishii, H., and P.-L. Lions. "Viscosity solutions of fully nonlinear second-order elliptic partial differential equations." Journal of Differential Equations 83 (1990): 26-78.

[12] Ivey, T. "Ricci solitons on compact three-manifolds." Differential Geometry and its Applications 3, no. 4 (1993): 301-7.

[13] Kleiner, B., and J. Lott. “Notes on Perelman's papers." (2006): preprint arXiv:math.DG/0605667.

[14] Koiso, N. “On rotationally symmetric Hamilton's equation for Kähler-Einstein metrics." Recent Topics in Differential and Analytic Geometry, 327-37. Advanced Studies Pure Mathematics 18. Boston, MA: Academic Press, 1990.

[15] Morgan, J., and G. Tian. "Ricci Flow and the Poincare Conjecture." (2006): preprint arXiv:math.DG/0607607.

[16] Ni, L. "Ancient solutions to Kähler-Ricci flow." Mathematical Research Letters 12, no. 5-6 (2005): 633-53.

[17] Ni, L., and N. Wallach. "On a classification of the gradient shrinking solitons." (2007): preprint arXiv:0710.3194

[18] Ni, L., and B. Wu. "Complete manifolds with nonnegative curvature operator." Proceedings of American Mathematical Society 135 (2007): 3021-8.

[19] Perelman, G. "The entropy formula for the Ricci flow and its geometric applications." (2002): preprint arXiv:math.DG/0211159.

[20] Perelman, G. "Ricci flow with surgery on three-manifolds." (2003): preprint arXiv:math.DG/0303109.

[21] Shi, W. X. "Deforming the metric on complete Riemannian manifolds." Journal of Differential Geometry 30 (1989): 223-301.

[22] Shi, W. X. "Ricci flow and the uniformization on complete noncompact Kähler manifolds." Journal of Differential Geometry 45 (1997): 94-220. 